\title{
THERMALIZATION IN INFRARED MULTIPLE PHOTON INDUCED REACTIONS. THE PRESSURE DEPENDENCE OF THE TRANS $\rightarrow$ CIS ISOMERIZATION OF CROTONITRILE
}

\author{
JOSEPH R. GUCKERT and ROBERT W. CARR \\ Department of Chemical Engineering and Materials Science, University of \\ Minnesota, Minneapolis, MN 55455-0132
}

(Received 24 August, 1989; in final form 8 December, 1989)

\begin{abstract}
The infrared multiple photon trans $\rightarrow$ cis isomerization of crotonitrile was studied over the pressure range 0.02 torr to 10 torr at fluences of approximately $4 \mathrm{~J} / \mathrm{cm}^{2}$ using collimated TEA $\mathrm{CO}_{2}$ laser radiation at $942 \mathrm{~cm}^{-1}$. Only minor quantities of fragmentation products were found at these conditions. At pressures of 0.1 torr or less, the trans form was completely converted to cis form by about 5000 laser pulses. On increasing the pressure a collisional regime was entered in which photostationary states were reached with fewer pulses and having smaller conversions than at low pressure. Both the number of pulses and the conversion decreased monotonically with increasing pressure. The tactic of driving the reaction in the exothermic direction gave evidence that the reaction does not occur solely by thermal isomerization even in the highly collisional 10 torr experiments, but that it still has a nonthermal component due to laser induced isomerization.
\end{abstract}

KEY WORDS: IR multiphoton, trans $\rightarrow$ cis isomerization, crotonitrile.

\section{INTRODUCTION}

When infrared multiple photon induced reactions are carried out at low pressures such that no collisions occur during the laser pulse, unimolecular reactions occur from species that are highly vibrationally excited and in vibrational disequilibrium with rotational and translational degrees of freedom. Various inter- and intramolecular selectivities may be exhibited, and reactions that have chemical equilibrium limited conversions when carried out thermally may be driven to completion. At high pressures, on the other hand, vibrational energy deposited in the sample may become collisionally thermalized. Thermalization is the point at which the deposited vibrational energy has reached equilibrium among all the degrees of freedom of the molecules in the irradiated volume. In the pressure region where the collisional frequency is sufficient to randomize the energy deposited during the laser pulse, the advantages of infrared laser-induced chemistry over thermal chemistry are lost.

From the practical viewpoint of maximizing the productivity of a multiphoton process, it is desirable to operate at high pressures. In practice the optimum pressure will be the result of a compromise between productivity and selectivity. It is important to characterize the transition from the collisionless regime to the thermal reaction. This article shows that this issue may be approached by investigating isom- 
erization reactions driven in the exothermic direction. A study of the trans $\rightarrow$ cis isomerization of crotonitrile is presented.

The temperature of a thermalized laser induced reaction may be calculated from Eq. (1), using the experimentally measured absorbed energy, $E_{\text {abs, }}$, and thermodynamic parameters.

$$
E_{\text {abs }}=\int_{T_{0}}^{T_{f}} \sum c_{v_{i}}(T) d T+\Delta H_{T_{0}}^{0}
$$

The summation in the integral runs over all the species in the reaction volume, and $\Delta H_{T_{0}}^{0}$ is the standard heat of reaction at $T_{0}$. Equation (1) assumes the mixture is thermalized instantaneously after the laser pulse, and ignores any cooling of the irradiated volume, thus giving an upper limit to the final temperature.

With an estimate of $T_{f}$, a thermal reaction yield can be calculated and compared with the observed yield. This method is best suited to two channel reactions, since temperature can be calculated from the product ratio and Arrhenius parameters without knowledge of the reaction time. In the $\mathrm{NH}_{3}$ sensitized decomposition of cyclobutanone the calculated temperature (Eq. (1)) agreed well with the temperature derived from the ratio of the yields of ethylene and cyclopropane, indicating a thermalized mixture. ${ }^{1}$ These results differ from the irradiation of pure cyclobutanone up to pressures of 10 torr, where estimates of the temperature based on Eq. ((1) were $100^{\circ} \mathrm{C}$ hotter than predicted by the ratio of the yields. ${ }^{2}$ The laser induced decomposition of $\mathrm{HCClF}_{2}$ has been studied at 60 torr, where hundreds of collisions occurred during the laser pulse. ${ }^{3}$ However, the $\mathrm{CF}_{2}$ yields from the decomposition of $\mathrm{HCClF}_{2}$ were orders of magnitude greater than could be accounted for thermally. It was concluded that the $\mathrm{HCClF}_{2}$ vibrational temperature was not in equilibrium with the ambient gas temperature during the reaction.

Another approach employed in thermalization studies involves the comparative rate technique. A nonabsorbing species which undergoes a unimolecular reaction is mixed with the reactant. Upon irradiation of the mixture, any observed reaction of the added species results from energy transfer from the excited reactant. Since the reaction time and temperature history of each species is identical, knowledge of these pararmeters is unnecessary. Furthermore, if the activation energies are similar for both the ratio of their yields from a purely thermal reaction will be temperature independent. Thermalization is assumed when the yields from the laser-induced reaction agree with the thermally predicted ratio.

Application of the comparative rate technique in the multiphoton reaction of $\mathrm{CH}_{3} \mathrm{CF}_{3}$ showed that thermalization occurred at pressures of 1-2 torr. ${ }^{4}$ In the multiphoton reaction of ethyl acetate, several investigators used isopropyl bromide as a thermal monitor. In one of these studies, the thermal limit was approached at 50 torr; ${ }^{5}$ whereas in another study thermalization was found to occur at 20 torr. ${ }^{6}$ This discrepancy can be due to different fluences used in the two experiments. In a more recent study of the ethyl acetate/isopropyl bromide system, a thermal contribution to the yield appeared at pressures as low as 0.100 torr. $^{7}$

The discrepancies in the point of thermalization can be due to differences in the 
experimental systems, such as the reactant or the laser beam fluences, or to shortcomings of the two techniques. The major shortcoming of Eq. (1) is the result of ignoring cooling. Cooling of the irradiated volume by a combination of shock, conduction, and/or diffusion could create a complex temporal temperature profile which would affect calculation of thermal reaction yields. On the other hand, the comparative rate technique might be influenced by rapid $V-V$ transfer between the reactant and standard. If this $\mathrm{V}-\mathrm{V}$ transfer is sufficiently rapid, then the standard may be vibrationally pumped and react before $\mathrm{V}-\mathrm{R} / \mathrm{T}$ equilibrium occurs. Another problem can arise if the standard becomes resonant with the laser radiation upon collisional excitation. It would then be able to undergo secondary photolysis during the laser pulse, again leading to erroneous conclusions on the extent of thermalization.

Here we show that another test of thermalization can be provided by studying exothermic reactions. At low pressure, both endothermic and exothermic reactions can give large, nonequilibrium yields via multiple photon excitation. At higher pressures, however, it is not possible to unambiguously determine whether high yields from endothermic reactions are due to a laser induced reaction or to thermalization, since in an endothermic reaction, the thermal equilibrium conversion increases with increasing temperature. However, for an exothermic reaction, the thermal equilibrium conversion decreases with increasing temperature. Thus, the multiple photon reaction yield should be large at low pressures where the reaction is clearly laser induced, and should decrease with increasing pressure. As the pressure is increased, collisions will deactivate the laser excited reactant, resulting in a decrease in the conversion. Eventually, the pressure will be sufficiently high to thermalize the mixture, and the temperature can be calculated from the measured extent of reaction and thermodynamic data. These experiments must be carried out with a lage number of laser pulses, such that yields become independent of the number of pulses.

Good candidates for this method are laser-induced isomerizations. The advantages are two-fold. First, the thermodynamic parameters for many isomerization reactions are in a range which give a measurable decrease in the equilibrium conversion between 300 and $1500^{\circ} \mathrm{K}$. Such temperatures can exist in laser-induced reactions. Secondly, an isomerization reaction does not affect the pressure in a fixed volume reactor. With a decomposition reaction, the pressure rise would complicate the analysis of the thermalization process.

In this work, the infrared multiphoton isomerization of trans-crotonitrile (TCN) is employed to investigate the above ideas. The thermal cis-trans isomerization of cis-crotonitrile (CCN) has been investigated by Butler and McAlpine ${ }^{8}$ and by Marley and Jeffers. ${ }^{9}$ The Marley and Jeffers rate constant, given in Arrhenius form in Eq. (2) for the trans $\rightarrow$ cis direction, is preferred for reasons stated in Ref. [9].

$$
\log k(T)\left(s^{-1}\right)=13.2-58,000 / 2.3 \mathrm{RT}
$$

Butler and McAlpine ${ }^{8}$ reported experimentally determined equilibrium cis/trans ratios from 303 to $455^{\circ} \mathrm{C}$, from which they derived (for cis $\rightarrow$ trans) 


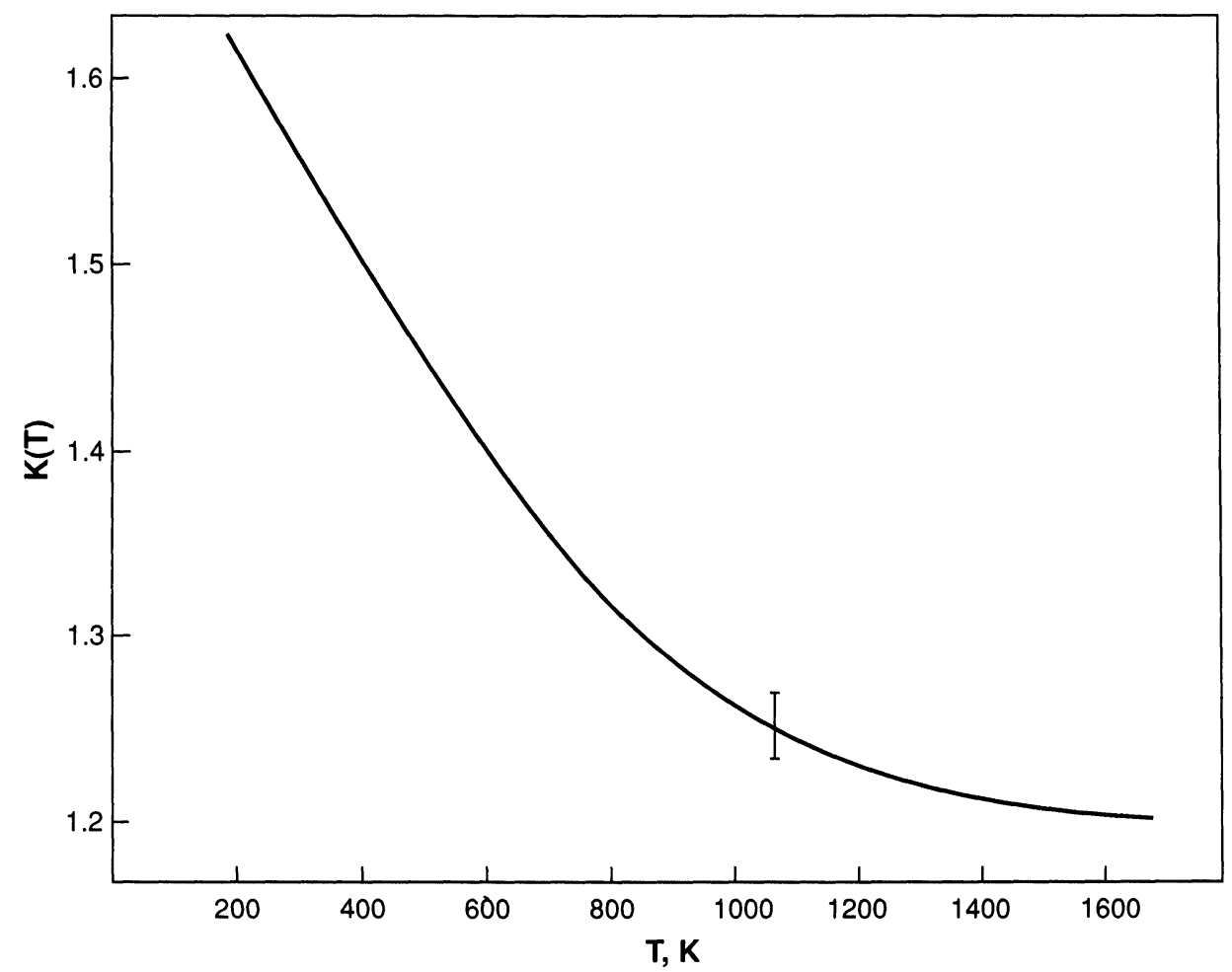

Figure 1 Equilibrium constant for $\mathrm{TCN} \rightleftharpoons \mathrm{CCN}$, calculated from spectroscopic data ${ }^{10,11}$ as a function of temperature.

$\Delta H^{\circ}=0.17 \pm 0.12 \mathrm{kcal} / \mathrm{mol}$ and $\Delta S^{\circ}=0.39 \pm 0.19 \mathrm{cal} / \mathrm{mol} \mathrm{K}$. Corresponding values of these quantities, calculated from the vibrational spectrum ${ }^{10}$ by the method of Pitzer and Hollenberg, ${ }^{11}$ are $0.259 \mathrm{kcal} / \mathrm{mol}$ and $-0.211 \mathrm{cal} / \mathrm{mol} \mathrm{K}$. The trans $\rightarrow$ cis experimental equilibrium constant is 1.39 at $673 \mathrm{~K},{ }^{8}$ while the statistical value is 1.38 . The equilibrium constant for trans $\rightarrow$ cis, calculated from the statistically derived thermodynamic functions of Ref. [10] is plotted $v s$. T in Figure 1. These values are used in this work since they can be reliably computed at the temperatures of interest, while the data of Ref. [8] would require extrapolation to temperatures greater than $750 \mathrm{~K}$.

The infrared spectra of cis and trans-crotonitrile between $900-1100^{-1}$ are displayed in Figure 2. The major absorption feature of CCN centered at $954 \mathrm{~cm}^{-1}$ is an unsymmetrical bending (out of plane) of the $\mathrm{HCCH}$ group. In TCN the absorption feature at $957 \mathrm{~cm}^{-1}$ is produced by a similar displacement of the $\mathrm{HCCH}$ group. ${ }^{10}$

In a previous study, both cis and trans-crotonitrile were irradiated at 931, 951 and $978 \mathrm{~cm}^{-1}$. At each of these frequencies, the irradiation of 0.10 torr of trans yielded a steady state value greater than $95 \%$ for cis. Irradiation of cis produced a minor isomerization to trans. ${ }^{12}$ 

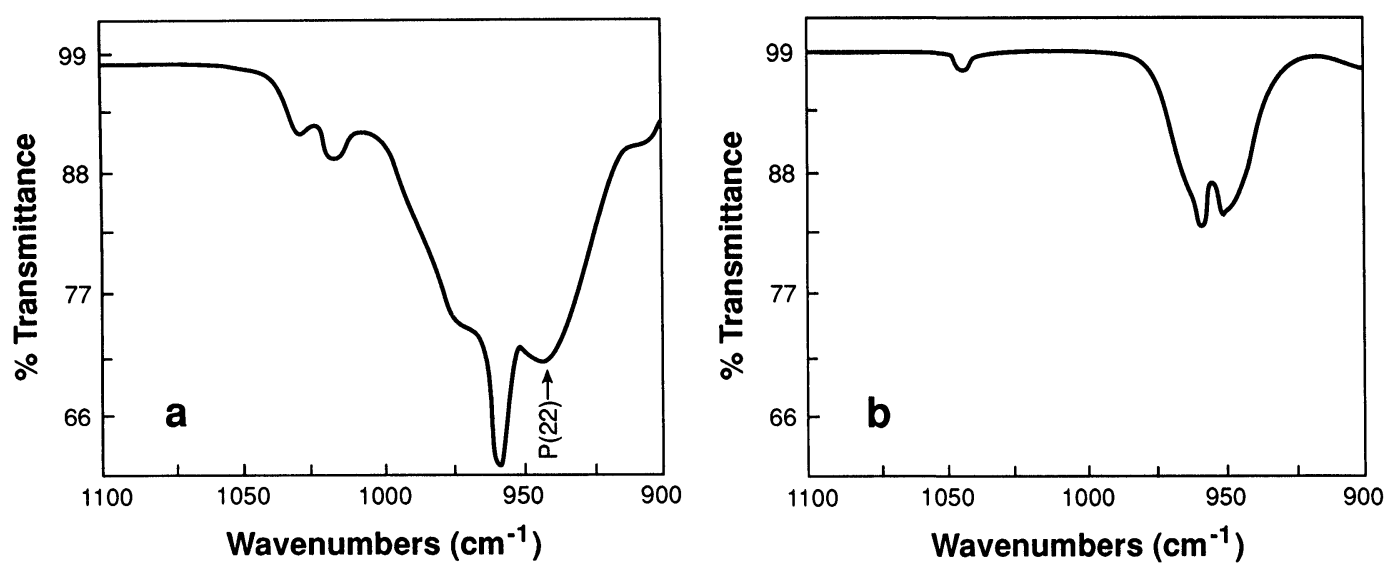

Figure 2 (a) FTIR spectrum of TCN, $p=100$ torr, $10 \mathrm{~cm}$ path length. (b) FTIR spectrum of CCN, $p=10$ torr, $10 \mathrm{~cm}$ path length.

\section{EXPERIMENTAL}

A mixture of cis and trans-crotonitrile was purchased from Pfaltz and Bauer. Separation of the isomers was performed by preparative gas chromatography, using a $0.9 \mathrm{~m}$ Porapak $\mathrm{Q}$ column at $130^{\circ} \mathrm{C}$. After separation, the isomers were identified by FTIR spectrometry. Further GC analysis showed the cis sample to be $99.8 \%$ pure (the impurity was not identified), while the trans sample contained $\sim 7 \%$ of the cis isomer.

Photolyses were done in $2.5 \mathrm{~cm}$-diameter Pyrex cells, either 4 or $8 \mathrm{~cm}$ long having $\mathrm{NaCl}$ end windows. Gas handling was performed on a conventional vacuum apparatus $\left(10^{-5}\right.$ torr) equipped with Kontes Teflon-glass valves. All pressures were measured with either an ionization gauge or MKS capacitance manometer.

Samples were irradiated at $942 \mathrm{~cm}^{-1}\left[\mathrm{P}(22)\right.$ line] with a Lumonics $102 \mathrm{TEA} \mathrm{CO} \mathrm{CO}_{2}$ laser. The laser pulse consisted of a $110 \mathrm{~ns}$ FWHM spike, followed by a low intensity tail which extended for $\sim 2 \mu \mathrm{sec}$. The tail contained approximately $60 \%$ of the total energy. The laser beam intensity was increased with a condensing telescope, constructed from $\mathrm{NaCl}$ lenses (f.l. $=20 \mathrm{~cm}$ and $-7.5 \mathrm{~cm}$ ). The divergence of the resulting beam was significant; the fluence decreased $\sim 20 \%$ over a length of $8 \mathrm{~cm}$. A $5.16 \mathrm{~mm}$ diam-aperture placed after the telescope defined the beam entering the reaction cell. The energy of the laser was normally adjusted to produce a fluence of $4.6 \pm 0.2 \mathrm{~J} / \mathrm{cm}^{2}$ inside the front window of the cell. Energy measurements were made with a Scientech calorimeter and power meter.

In several experiments, energy deposition was measured using differential calorimetry. A beamsplitter, placed before the condensing telescope, diverted a portion of the laser beam to a second Scientech calorimeter. The fluence of the transmitted beam was $4.3 \pm 0.1 \mathrm{~J} / \mathrm{cm}^{2}$. Also, in order to minimize fluence variations due to beam divergence, cell lengths were reduced from $8 \mathrm{~cm}$ to $4 \mathrm{~cm}$ for these experiments. 


\section{RESULTS}

Irradiation of TCN formed $\mathrm{CCN}$ as the major product. While decomposition products were observed, since the sum of their yields was usually less than $1 \%$ at the fluences $\left(\sim 4 \mathrm{~J} / \mathrm{cm}^{2}\right)$ used in routine experiments they were not positively identified.

The laser wavenumber dependence of $\mathrm{CCN}$ yields was investigated at 0.05 torr, where on average less than one collision occurs during the laser pulse, and approximately $4 \mathrm{~J} / \mathrm{cm}^{2}$. Yields of CCN per $10^{3}$ pulses from these experiments are given in Table 1. At fixed wavenumber, yield is seen to be somewhat sensitive to fluence. For the asterisked entry the laser changing voltage was about $2 \mathrm{kV}$ less than the usual 34 $\mathrm{kV}$ setting. While the fluence was the same as in an experiment at the higher charging voltage, the pulse width was slightly longer. The significantly smaller yield observed may be due to an intensity effect. This effect was observed in other experiments. This aspect of the work was not pursued, and care was taken in subsequent experiments to set laser power levels and measured fluences reproducibly.

Cis-crotonitrile was irradiated at $942 \mathrm{~cm}^{-1}$ with fluences and at pressures similar to those used for TCN photolysis. Although CCN absorbs $942 \mathrm{~cm}^{-1}$ radiation, no isomerization to TCN was detected in 0.05 and 5.0 torr samples after 2000 pulses.

The $942 \mathrm{~cm}^{-1}$ laser line was selected for investigation of the pressure dependence of the limiting CCN yields. At all pressures between 0.01 torr and 10 torr it was found that the $\mathrm{CCN}$ yields increased with increasing number of laser pulses to a pressure dependent asymptote. The upper pressure was limited to 10 torr in these experiments since the vapor pressure of $\mathrm{TCN}$ is 14.2 torr at $22^{\circ} \mathrm{C}$. Table 2 shows the results from experiments at 5 torr and 10 torr. At 5 torr the $\mathrm{CCN}$ yield appears to reach a limiting value at about 2000 pulses, while at 10 torr the limit is reached at 1000 pulses. The average percent $\mathrm{CCN}$ yield for the 1985 through 5000 pulse experiments at 5 torr is $56.5 \pm 0.5$, and for the 1000 and 2000 pulse experiments at 10 torr it is $56.0 \pm 0.3$. Similar experiments at 1 torr gave a limiting CCN percent yield of $65.4 \pm 1.9$ averaged over 4000 to 7000 pulse experiments. The CCN/TCN ratio also reached a constant value. The average limiting values of $\mathrm{CCN} / \mathrm{TCN}$ at 10,5 and 1 torr are $1.28 \pm 0.02,1.36 \pm 0.04$ and $1.94 \pm 0.19$, respectively.

Table 1 Laser wavenumber dependence of $\mathrm{CCN}$ yields at 0.05 torr

\begin{tabular}{lll}
\hline$\tilde{v}\left(\mathrm{~cm}^{-1}\right)$ & Fluence $\left(\mathrm{J}^{\left.-\mathrm{cm}^{2}\right)}\right.$ & $\%$ CCN (per 10 Pulses) \\
\hline 931 & 3.98 & 19.0 \\
& 4.14 & 20.7 \\
942 & 3.99 & 25.6 \\
& 4.04 & 26.3 \\
& 4.28 & 61.1 \\
951 & $4.28^{\mathrm{a}}$ & 32.6 \\
& 4.04 & 25.5 \\
954 & 4.53 & 56.7 \\
& 4.04 & 23.9 \\
978 & 4.29 & 43.0 \\
\hline
\end{tabular}

${ }^{a}$ Laser charging voltage $2 \mathrm{kV}$ smaller than other experiments in the table. 
Table 2 Dependence of $942 \mathrm{~cm}^{-1} \mathrm{CCN}$ yield upon number of laser pulses

\begin{tabular}{lllll}
\hline p(torr) & \# pulses & Fluence $\left(\mathrm{J} / \mathrm{cm}^{2}\right)$ & $\%$ CCN & CCN/TCN \\
\hline 5.0 & 100 & 4.5 & 43.0 & 0.76 \\
& 500 & 4.5 & 52.3 & 1.10 \\
& 1,000 & 4.4 & 54.5 & 1.20 \\
& $1,985^{\mathrm{a}}$ & 4.3 & 56.2 & 1.28 \\
& $2,000^{\mathrm{a}}$ & 4.4 & 55.9 & 1.27 \\
& $2,034^{\mathrm{a}}$ & 4.3 & 56.2 & 1.29 \\
& $3,000^{\mathrm{a}}$ & 4.3 & 56.9 & 1.33 \\
10.0 & $5,000^{\mathrm{a}}$ & 4.3 & 57.3 & 1.35 \\
& 100 & 4.4 & 45.8 & 0.85 \\
& 500 & 4.5 & 54.3 & 1.19 \\
& $1,000^{\mathrm{a}}$ & 4.5 & 55.9 & 1.28 \\
& $1,000^{\mathrm{a}}$ & 4.3 & 55.6 & 1.25 \\
& $2,000^{\mathrm{a}}$ & 4.4 & 56.5 & 1.31 \\
\hline
\end{tabular}

The limiting CCN yields as a function of pressure are displayed in Figure 3. It is readily seen that at pressures below about 0.1 torr, the isomerization is driven to completion. The $100 \%$ yields required about 5000 laser pulses, after which only traces of TCN remaned. At higher pressures, it is no longer possible to completely convert TCN, the limiting yields of TCN decreasing monotonically with increasing pressure.

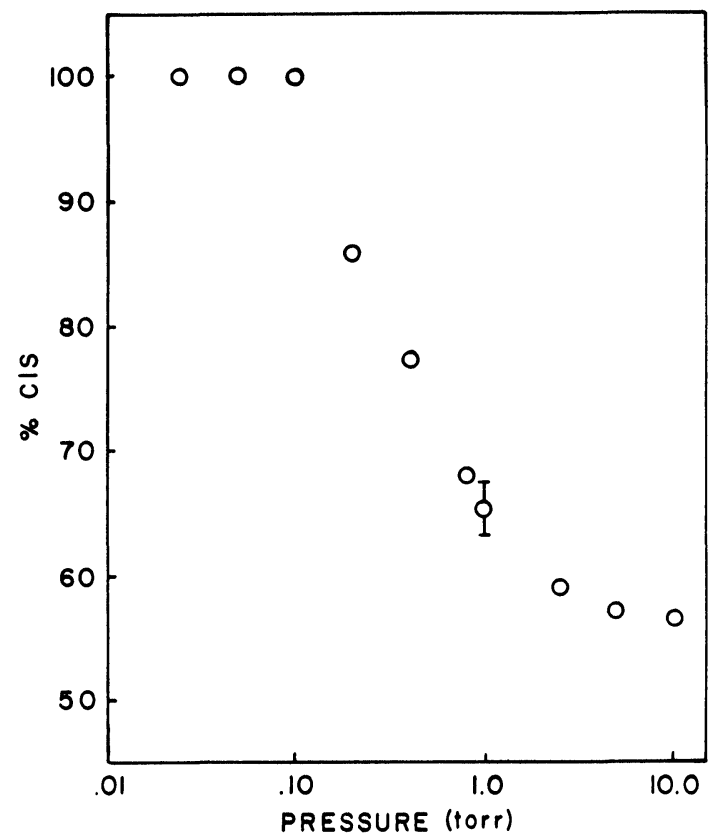

Figure 3 Pressure dependence of limiting photostationary state yields of $\mathrm{CCN} v s$. pressure. 


\section{DISCUSSION}

The results can perhaps best be understood by starting with the following simplified mechanism.

$$
\begin{gathered}
\mathrm{TCN}+\mathrm{nh} v \longrightarrow \mathrm{TCN}^{*} \\
\mathrm{TCN}^{*} \longleftrightarrow \mathrm{CCN}^{*} \\
\mathrm{TCN}^{*}+\mathrm{M} \longrightarrow \mathrm{TCN}+\mathrm{M} \\
\mathrm{CCN}^{*}+\mathrm{M} \longrightarrow \mathrm{CCN}+\mathrm{M}
\end{gathered}
$$

Step (1) lumps the multiple photon excitation of TCN, with its attendant complexities, into a single step since these experiments contain no useful information on excitation mechanisms. Asterisk represents vibrational levels above the critical energy for isomerization. Step (2) indicates that the isomerization is expected to be reversible at those excitation energies, while (3) and (4) indicate collisional deactivation of both hot isomers to energies below the critical energy. Deactivation is written as a single step (strong collision) mechanism since this work is not concerned with details of energy transfer. $\mathrm{M}$ is a collision partner, which here is either cold TCN or $\mathrm{CCN}$.

The 4-step mechanism serves to explain the low pressure results. From Figure 3, the isomerization can be carried out to completion at pressures of approximately 0.1 torr or less. The collision frequency of $\mathrm{TCN}^{*}$ or $\mathrm{CCN}^{*}$ at 0.1 torr is approximately 1 $\mu \mathrm{s}^{-1}$, assuming a hard-sphere collision diameter of $5.5 \times 10^{-1} \mathrm{~cm}$. Thus, during the laser pulse very few collisions occur, the asterisk states are populated by laser excitation, not by collisions, and the reaction may in that sense be characterized as laser induced. Since pure CCN does not undergo laser induced isomerization the back reaction must not occur unless $\mathrm{CCN}$ that is partially deactivated can absorb laser radiation while in the quasi-continuum of vibrational states. If this occurs, complete conversion can still be attained if either the rate of absorption of radiation by TCN is greater than by $\mathrm{CCN}^{*}$, or if equilibrium is established in step (2) and the vibrational state density of $\mathrm{CCN}^{*}$ exceeds that of $\mathrm{TCN}^{*}$ at each energy above the critical energy. In any case it is not necessary to invoke reversibility to explain the results.

At pressures greater than about 0.1 torr, it is no longer possible to drive the reaction to completion, and the 4-step mechanism must be modified. In the range 0.1 to 1.0 torr, approximately 2 to 20 collisions occur during the laser pulse. Although collisions undoubtedly play a role, there are an insufficient number for the system to be thermalized over this pressure range. This is corroborated by comparing observed photostationary $\mathrm{CCN}$ to TCN ratios with equilibrium constants. If the system were thermalized the temperature calculated from the equilibrium constant $\mathrm{K}(\mathrm{T})=\mathrm{CCN} / \mathrm{TCN}$ would be less than $300 \mathrm{~K}$, even at 1 torr. However, at $300 \mathrm{~K}$ the thermal isomerization rate is orders of magnitude too small to explain the observed 
yields. Since a photostationary state is reached, it is clear that the rate of the reverse reaction must become equal to the rate of the forward reaction during each laser pulse at a conversion that decreases with increasing pressure. Since isomerization of pure cold CCN was not observed, it must be that the back reaction involves vibrationally hot $\mathrm{CCN}$. The only possibilities seem to be absorption of laser radiation, or perhaps an energy pooling mechanism. Since it was not important to diagnose the loss of selectivity for the purpose of this communication, further experiments were not done in this pressure regime.

At higher pressures, there is further loss of selectivity, and of course, many more collisions. At 5 torr, on average more than 100 collisions occur during the laser pulse. This suggests that the system may be thermalized or close to it. However, it is not clear that a thermal reaction is occurring. It is possible that collisional deactivation of $\mathrm{TCN}^{*}$ by $\mathrm{CCN}$ becomes sufficiently rapid at higher pressures to quench the reaction. At low conversions, some collisions which occur during the laser pulse are probably between $\mathrm{TCN}^{*}$ molecules. These collisions can result in up-pumping of one of the colliders, thereby enhancing the reaction rate. However, at larger conversion, collisions involving $\mathrm{TCN}^{*}$ and cold cis molecules become more likely. This possibility is supported by experiments where the addition of 10 torr of $\mathrm{N}_{2}$ to 1 torr of TCN completely quenched the reaction.

In order to further investigate thermal effects, energy deposition studies were made using differential calorimetry. Since the addition of the beamsplitter slightly altered the fluence and geometry of the beam, additional experiments were performed to reestablish the steady cis/trans ratio. These results are listed in Table 2 . Using the runs marked with asterisks, average cis/trans ratios of $1.31 \pm 0.03$ and $1.28 \pm 0.03$ are calculated for 5 and 10 torr respectively. These values ae slightly lower (4\%) than the results plotted in Figure 3.

The results of the energy deposition studies are reported in Table 3. Included in this table are measurements that were made during the initial and final pulses of an experiment. The experiments at higher fluences than reported in Table 2 were performed after the installation of new $\mathrm{NaCl}$ lenses in the condensing telescope. The replacement of the lenses improved the beam quality, including the reduction of the divergence and an increase in the fluence. The large uncertainty in the values reflect

Table 3 Estimated temperatures in $942 \mathrm{~cm}^{-1}$ irradiations

\begin{tabular}{|c|c|c|c|c|c|c|c|}
\hline \multirow[b]{2}{*}{$p($ torr $)$} & \multirow[b]{2}{*}{$\mathrm{fl}\left(\mathrm{J} / \mathrm{cm}^{2}\right)$} & \multirow[b]{2}{*}{$E_{\text {abs }}(J)$} & \multirow[b]{2}{*}{ \# pulses } & \multirow[b]{2}{*}{$\% T C N$} & \multirow[b]{2}{*}{$C C N / T C N$} & \multicolumn{2}{|l|}{$T(K)$} \\
\hline & & & & & & $E q .(1)$ & cis/trans $K(T)$ \\
\hline 5.00 & $\begin{array}{l}4.30 \\
4.94\end{array}$ & $\begin{array}{l}0.034 \pm 0.011 \\
0.040 \pm 0.013 \\
0.036 \pm 0.011 \\
0.033 \pm 0.012\end{array}$ & $\begin{array}{l}\text { (a) } \\
1985 \\
\text { (a) } \\
1,000\end{array}$ & $\begin{array}{l}93.3 \\
43.8 \\
93.3 \\
41.2\end{array}$ & $\begin{array}{l}\overline{1.28} \\
\overline{1.40}\end{array}$ & $\begin{array}{l}\sim 1,350 \\
1,500 \pm 500 \\
\sim 1400 \\
1,350 \pm 500\end{array}$ & $\begin{array}{l}\overline{9} 30 \pm 100 \\
\overline{6} 20 \pm 60\end{array}$ \\
\hline 10.00 & $\begin{array}{l}4.30 \\
4.91\end{array}$ & $\begin{array}{l}0.078 \pm 0.013 \\
0.060 \pm 0.010 \\
0.077 \pm 0.011 \\
0.057 \pm 0.010\end{array}$ & $\begin{array}{l}\text { (a) } \\
1000 \\
\text { (a) } \\
500\end{array}$ & $\begin{array}{l}93.3 \\
44.4 \\
93.3 \\
41.7\end{array}$ & $\begin{array}{l}\overline{1.25} \\
\overline{1.36}\end{array}$ & $\begin{array}{l}\sim 1,500 \\
1,250 \pm 200 \\
\sim 1,500 \\
1,250 \pm 200\end{array}$ & $\begin{array}{l}\overline{1,030} \pm 200 \\
\overline{700 \pm 65}\end{array}$ \\
\hline
\end{tabular}

(a) Absorbed energy averaged over the first few pulses. 
the limit of the calorimeter accuracy. The transmission of the gas samples ranged from 0.90 to 0.95 and were able to be measured to within \pm 0.02 .

Table 3 also includes two estimates of the temperature. One of the estimates is based on the absorbed energy and Eq. (1). These temperatures must be regarded as approximate, due to the extrapolation of the thermodynamic data above $1000^{\circ} \mathrm{K}$ and the error in $E_{\text {abs. }}$.

The other temperature estimate is based on the $\mathrm{CCN} / \mathrm{TCN}$ ratio and the thermodynamic equilibrium constant. The $\mathrm{CCN} / \mathrm{TCN}$ ratios were determined by GC analysis, and are also displayed in Table 2 . While the ratios at $4.3 \mathrm{~J} / \mathrm{cm}^{2}$ agree well with previous results, the ratios at $4.9 \mathrm{~J} / \mathrm{cm}^{2}$ (produced by the new telescope optics) are slightly larger after fewer pulses.

As can be seen from Figure 1, the rate of decrease of $K(T)$ slows as $T$ increases. Since the CCN/TCN ratio is reproducible to $+2 \%$ for values in the vicinity of 1.3 to 1.2 (error bar shown in Figure 1), the uncertainty in $T$ increases with decreasing CCN/TCN. The uncertainty of $T$ is given in the last column of Table 3. Although the error limits by the two kinds of temperature estimates overlap in the $4.3 \mathrm{~J} / \mathrm{cm}^{2}$ experiments, the mean $T$ from CCN/TCN measurements is lower at both 5 and 10 torr than from the $E_{\text {abs }}$ measurements. At $4.9 \mathrm{~J} / \mathrm{cm}^{2}, T$ from CCN/TCN is clearly lower than from $E_{\text {abs }}$. Furthermore, Table 3 shows that the experiments performed at $4.9 \mathrm{~J} / \mathrm{cm}^{2}$ result in larger $C C N / T C N$ ratios than at $4.3 \mathrm{~J} / \mathrm{cm}^{2}$ even though the energy depositions are similar. If the mixture were completely thermalized, CCN/TCN would depend only upon $E_{\text {abs }}$.

Quenching or secondary absorption by cis-crotonitrile can be discounted as possible explanations for the difference in the estimated temperatures. Both of these processes could limit the conversion of TCN, and therefore prevent increases in CCN/TCN. While the role of quenching is straightforward, further absorption by cis would replenish trans by either a multiphoton driven reaction, or by the further heating of a thermalized mixture.

It is possible that cooling of the laser heated gas causes the mean temperature to be lower than predicted by Eq. (1). The cooling process involves a complex mechanism, resulting from a combination of shock waves, conduction and diffusion. ${ }^{13,14}$ The mixture is partially cooled by the rarefaction wave accompanying the outgoing shock wave originating at the boundary of the column of heated gas and the surrounding cool gas. The time scale for this process is a few microseconds. ${ }^{15}$ Final cooling by thermal conductivity is completed on the order of milliseconds. However, a reaction time of about $3 \mathrm{~min}$ would be required for the observed $0.2 \%$ yield $/$ pulse at $700^{\circ} \mathrm{K}$, while a $1 \mathrm{msec}$ reaction time would require $T=975 \mathrm{~K}$, clearly too high to be compatible with the measured $K(T)$, even if fairly large uncertainties in its value exist. Thus, the reaction proceeds too slowly to account for the establishment of chemical equilibrium at that temperature.

If the measured $\mathrm{CCN} / \mathrm{TCN}$ results from a completely thermal reaction that is rate limited in the sense that the reaction is not fast enough to establish chemical equilibrium at a specified temperature, then $\mathrm{CCN} / \mathrm{TCN}$ would be smaller than the expected equilibrium ratio at that temperature. The result of assuming thermal and chemical equilibrium would be to overestimate $T$ from use of Figure 1. Thus, $T$ 
from CCN/TCN would be higher than from $E_{\text {abs, }}$, in contrast with the lower $T$ found.

The observations at 5 torr and 10 torr are consistent with the expected behavior if the system is not completely thermalized. In this case, $\mathrm{CCN} / \mathrm{TCN}$ would be greater than would be obtained in a system at thermal equilibrium due to the laser induced isomerization. The result of mistakenly assuming thermal and chemical equilibrium and employing Figure 1 would be to underestimate $T$. This can then account for the direction of the temperature discrepancy in Table 3.

The detailed mechanism of the isomerization in the 5-10 torr range must be complicated, with collisional energy transfer of all varieties $(\mathrm{V}-\mathrm{V}, \mathrm{V}-\mathrm{T}, \mathrm{V}-\mathrm{T} / \mathrm{R})$ occurring in competition with the multiple photon dynamics. Modification of the 4-step scheme to accommodate this complexity would be required, but is not attempted here. The evidence indicates that even at 10 torr there is real competition, and that collisional processes are not sufficiently fast to thermalize the system before laser excitation to $\mathrm{TCN}^{*}$ can contribute to the yield of $\mathrm{CCN}$.

The pressure at which thermalization is complete, and the observed reaction is a thermal isomerization or decomposition, depends upon many factors. Important quantities are the laser fluence, the cross-section for absorption of radiation and the Arrhenius parameters. It is expected that rather wide variability exists in the pressure range in which thermalization occurs in infrared multiple photon reactions.

\section{References}

1. C. Steel, V. Starov, R. Lee, P. John and R. G. Manson, Chem. Phys. Let. 62, 121 (1979).

2. P. John, M. R. Humphries, R. G. Harrison and P. G. Harper, J. Chem. Phys. 79, 1353 (1983).

3. R. C. Slater and J. H. Parks, Chem. Phys. Let. 60, 275 (1979).

4. T. H. Richardson and D. W. Setser, J. Phys. Chem. 81, 2301 (1977).

5. D. Gutman, W. Braun and W. Tsang, J. Chem. Phys. 67, 4291 (1977).

6. W. C. Danen, W. D. Munslow and D. W. Setser, J. Am. Chem. Soc. 99, 6961 (1977).

7. W. C. Danen, V. C. Rio and D. W. Setser, J. Am. Chem. Soc. 104, 5431 (1982).

8. J. N. Butler and R. D. McAlpine, Can. J. Chem. 41, 2487 (1963).

9. W. M. Marley and P. M. Jeffers, J. Phys. Chem. 79, 2085 (1975).

10. J. R. Durig, C. K. Tong, C. W. Hawley and J. Bragin, J. Phys. Chem. 75, 44 (1971).

11. K. S. Pitzer and J. C. Hollenberg, J. Am. Chem. Soc. 76, 1493 (1954).

12. P. P. Teng, E. Weitz and F. D. Lewis, J. Am. Chem. Soc. 104, 5518 (1982).

13. P. W. Fairchild, G. P. Smith and D. R. Crosley, J. Chem. Phys. 79, 1795 (1983).

14. P. W. Fairchild, G. P. Smith and D. R. Crosley, 19th Symposium (International) on Combustion, The Combustion Institute, Pittsburgh, p. 107 (1982).

15. J. R. Guckert and R. W. Carr, J. Phys. Chem. 90, 4286 (1986). 